\title{
Publisher's Note: Achiral tilted domain walls in perpendicularly magnetized nanowires [Phys. Rev. B 95, 180406(R) (2017)]
}

\author{
B. Boehm, A. Bisig, A. Bischof, G. Stefanou, B. J. Hickey, and R. Allenspach \\ (Received 1 June 2017; published 7 June 2017)
}

DOI: 10.1103/PhysRevB.95.219903

This paper was published online on 25 May 2017 with an error in the Acknowledgments. The last sentence in the Acknowledgments on page 4 should read as "The research leading to these results has received funding from the European Union Seventh Framework Programme (FP7-People-2012-ITN) under Grant Agreements No. 316657 (SpinIcur) and No. 608031 (Wall)." The Acknowledgments have been corrected as of 2 June 2017. The Acknowledgments are correct in the printed version of the journal. 